\title{
Impact de la formation en français sur objectifs spécifiques sur la compétence discursive de l'apprenant scientifique marocain : cas de l'Ecole Nationale d'Agriculture de Meknès
}

Jamila TAMESNA, Ecole Nationale d'Agriculture, Meknès-Maroc

Le Maroc a connu depuis 2003 un ensemble de réformes universitaires d'envergure, dont l'enjeu majeur étant de rentabiliser au mieux l'enseignement supérieur. Ces réformes ont été consolidées par les orientations du plan d'urgence 2009-2012, où l'on accorde une place privilégiée à l'enseignement des langues en soulignant leur importance dans l'acquisition du savoir et du savoir-faire universitaires.

Par ailleurs, la question sociolinguistique au Maroc est une des problématiques les plus difficiles car les signes de ce que l'on peut appeler à l'évidence une crise linguistique sont visibles. On peut évoquer en l'occurrence l'absence d'une politique linguistique globale en matière de planification et d'aménagement linguistique, le problème de la langue d'enseignement, de l'arabisation, du statut réel de l'arabe standard et de son rapport avec l'arabe dialectal, le statut de l'amazighe et sa reconnaissance en tant que langue officielle à côté de l'arabe dans la nouvelle constitution de 2011. A cela s'ajoute l'avenir des langues étrangères et plus particulièrement le français qui serait potentiellement concurrencé par l'anglais considéré comme langue d'ouverture sur le monde occidental, sur la science et la technologie.

Ainsi, le processus d'arabisation des matières scientifiques entrepris au Maroc au niveau des enseignements fondamental et secondaire et le maintien du français dans le supérieur technico-scientifique ont nécessité le recours à de nouvelles approches de l'enseignement de la langue française, dont les plus marquantes sont les approches communicative et fonctionnelle. Pour cette dernière, il s'agit d'un français «adapté » qui se nourrit notamment du FOS (Français sur Objectifs Spécifiques), et du Cadre Européen Commun de Référence pour les Langues, adapté au contexte marocain. Cependant, les actions et les réformes entreprises n'ont pas atteint les objectifs assignés, et n'ont pas répondu de manière plus objective aux besoins des étudiants en matière de langue car les difficultés linguistiques subsistent. Suite à cet état de fait, nous avons voulu analyser l'impact de la formation en FOS sur la compétence textuelle et discursive de l'élève-ingénieur de l'Ecole Nationale d'Agriculture de Meknès. L'analyse de l'appropriation des faits grammaticaux et discursifs en langue étrangère soulève quelques questions théoriques. Doit-on renvoyer à des compétences distinctes les savoirs grammaticaux et discursifs en cours d'acquisition? 
L'acquisition grammaticale est-elle autonome par rapport à l'acquisition discursive ? Nous avons tenté d'apporter quelques éléments de réponse aux différents questionnements sus soulevés dans le cadre d'une recherche doctorale menée auprès du public marocain, à partir de notre terrain d'application.

\section{I-Présentation du contexte général de la recherche}

Le revirement méthodologique concernant l'enseignement/apprentissage du français qu'a connu le Maroc ces dernières années coïncide, d'une part, avec le réaménagement du paysage éducatif marocain, voire une redéfinition du statut de la langue française et de ses fonctions et d'autre part, avec le processus d'arabisation des disciplines scientifiques. En effet, le Maroc a connu depuis 2003 un ensemble de réformes universitaires d'envergure, lesquelles ont été consolidées par les orientations du plan d'urgence de 2009-2012 englobant plusieurs dispositifs, où l'on accorde une place privilégiée à l'enseignement des langues en soulignant leur importance dans l'acquisition du savoir et du savoir-faire universitaires. L'enjeu majeur de ces réformes étant de rentabiliser au mieux l'enseignement supérieur, et d'instaurer une adéquation entre le développement socio-économique et les enseignements universitaires.

Dans ce contexte d'arabisation touchant l'enseignement secondaire marocain, et le maintien du français dans le supérieur scientifique et technique, les enseignants des sciences dans les facultés et les institutions supérieures déploraient et continuent à déplorer le niveau de langue française de leurs étudiants. En effet, l'étudiant se trouve en rupture avec deux systèmes linguistiques différents (l'arabe standard et le français) marquant son passage de l'enseignement secondaire au supérieur. Rupture non sans répercussions sur les performances langagières de l'étudiant, se manifestant d'une part par la baisse du niveau de la langue d'enseignement qui associe la langue usuelle et la langue scientifique, et d'autre part, par les difficultés linguistiques rencontrées par ces étudiants pour poursuivre efficacement leurs études de spécialités, lesquelles continuent à être dispensées en français.

Pour remédier à cette situation paradoxale, plusieurs actions éducatives ont été entreprises en vue de réformer l'enseignement et d'améliorer le niveau linguistique des élèves qui accéderaient à l'enseignement supérieur : augmentation de l'horaire de français, du coefficient de la discipline à l'examen du baccalauréat, instauration d'heures d'activités scientifiques en français, formation de professeurs-traducteurs. A cela s'ajoute l'ensemble de projets de coopération franco-marocaine visant l'enseignement secondaire, ainsi que certaines écoles à caractère scientifique. C'est dans ce contexte qu'un cursus de formation modulaire en 
Français fonctionnel a été créé et inséré au cursus académique assuré à l'Ecole Nationale d'Agriculture de Meknès (ENAM). Cette dernière est un établissement public marocain d'enseignement agronomique, du développement rural et de la recherche scientifique, destiné à former en cinq ans des ingénieurs agronomes.

Ce cursus vise à renforcer le degré de maîtrise de la langue française des étudiants, à améliorer leur aptitude à suivre des études scientifiques, et à leur faire acquérir des savoirfaire précis indispensables en cours de formation (lecture active/analytique, prise de notes sur document écrit/oral, prise de parole, rédaction de rapports de stage et du mémoire de fin d'études, etc.). A noter que la pédagogie mise en œuvre est une pédagogie par objectifs et que l'enseignement adopté se caractérise par une récurrence des objectifs, afin de faire acquérir progressivement à l'étudiant les compétences ciblées. L'enjeu consiste à élaborer un dispositif le plus complet et le plus cohérent possible pour répondre aux besoins réels de notre public. Suite à ces données, la problématique formulée est la suivante : dans quelle mesure la création de ce cursus et l'adoption de cette nouvelle approche de l'enseignement de la langue française a pu résoudre la difficulté de la communication pédagogique soulevée dans le supérieur scientifique, combler les lacunes signalées par les professeurs de sciences et doter les étudiants de compétences adéquates?

Dans le cadre de cette problématique, nous nous proposons d'analyser et d'évaluer la compétence textuelle de l'élève-ingénieur agronome soumis à ce type d'enseignement de la langue et ce, à partir de corpus écrits recueillis auprès d'un échantillon de 62 étudiants de l'ENAM et appartenant à trois niveaux d'études différents, à savoir la $2^{\mathrm{e}}, 3^{\mathrm{e}}$ et $4^{\mathrm{e}}$ année. Notre objectif consiste à vérifier si les objectifs ciblés à travers cette formation ont été atteints, si les capacités développées convergent vers le profil linguistique requis pour un étudiant arabisant accédant à une formation scientifique en agronomie, et s'ils ont répondu aux besoins langagiers requis pour les disciplines de spécialité, notamment le mode d'organisation ou de structuration du savoir.

\section{II- Méthodologie}

Pour illustrer notre approche du niveau de la compétence textuelle réceptive et productive, nous avons opté pour le compte rendu, car pour ce dernier, l'étudiant doit faire preuve de la maîtrise d'une technique d'approche du texte, d'une compétence de lecture doublée d'une compétence d'écriture. La méthodologie adoptée repose sur trois outils d'investigation à savoir, le questionnaire, l'analyse de contenu du compte rendu d'un article de vulgarisation scientifique choisi comme support de travail, et l'analyse de contenu du 
tableau récapitulatif du même texte-support. Le questionnaire comporte cinq parties : chaque partie correspond à un savoir-faire dispensé en cursus de formation, laquelle partie est subdivisée en cinq items (a-b-c-d-e), dont le dernier constitue une question ouverte et correspond au recueil d'opinions. Pour le premier outil, nous proposons d'analyser le contenu des données recueillies par écrit en procédant d'abord à une analyse fréquentielle des réponses enregistrées par les enquêtés, ensuite au traitement statistique par le logiciel-tableur «MS Excel ». Cette approche nous a permis de calculer les paramètres de position et de dispersion, dont le premier permet d'avoir la moyenne de la variable «Utilité » pour chaque module composant le cursus de formation (lecture active, prise de notes sur document oral /sonore, prise de notes sur document écrit, production écrite, préparation de l'exposé), et de localiser l'échantillon moyen d'un niveau par rapport à un autre. Quant au second paramètre, il permet de calculer l'écart-type et le coefficient de variation afin d'évaluer les concentrations des réponses pour la même variable.

Les objectifs assignés au questionnaire consistent à :

- Evaluer l'utilité du programme de formation en français fonctionnel selon les trois niveaux précités, et sa contribution à la réalisation des tâches de l'étudiant relevant des enseignements de spécialité de l'ENAM ;

- Corréler les résultats qui ressortiront de l'analyse du questionnaire à ceux enregistrés à partir de l'analyse du contenu du corpus écrit collecté auprès du même échantillon et ce, en vue de vérifier l'impact de la formation sur l'amélioration des performances langagières et méthodologiques des étudiants.

Pour le second outil, nous proposons d'analyser les spécificités du discours produit en situation par les étudiants de l'ENAM, d'évaluer la compétence textuelle à travers ses deux composantes : réceptive et productrice en prenant en considération les deux niveaux de structuration du texte à savoir, le niveau micro-structurel et le niveau macro-structurel. Nous rappelons que pour notre analyse, nous nous sommes inspirés des théories de la grammaire des textes, l'analyse du discours et la grammaire textuelle, et des différents auteurs ayant approché l'analyse de textes ou de récits tels que Teun Van Dijk, Jean-Michel Adam, Heribert Rück et François Fontaine.

Les objectifs visés à travers l'analyse du contenu du compte rendu consistent à :

- Déceler la capacité de rétention et de transfert des acquis en situation de production écrite et en fonction des savoir-faire développés,

- Evaluer la capacité de l'étudiant à s'approprier les contenus pour les reformuler selon une démarche conceptuelle logique, 
- Procéder enfin à une évaluation comparative des productions langagières des trois niveaux testés en vue de dégager les différentes configurations textuelles possibles.

La démarche analytique de la compétence textuelle du public-cible s'appuie sur deux paramètres : le premier correspond à la superstructure du texte et vise à évaluer l'habileté de la lecture-compréhension et vérifier si l'étudiant a intégré les différentes pratiques de lecture dispensées lors du module réservé à cet effet, conduisant à leur mise en œuvre en vue d'une production écrite. Ainsi, l'étudiant est amené non seulement à mentionner la (es) stratégie (s) de lecture adoptée(s), mais à expliquer notamment les différentes entrées qui l'ont aidé à s'orienter dans sa lecture.

Le deuxième paramètre est composé de deux volets au travers desquels nous avons évalué l'habileté de l'écriture-production des étudiants testés. Le premier volet est organisé autour de trois blocs de sens ayant trait à la dimension textuelle à savoir, la situation introductive (problématisation), le développement (analyse et élucidation des éléments du problème) et la conclusion. L'objectif consiste à analyser l'aptitude de l'étudiant à identifier le schéma de base du texte initial, à développer les idées directrices et à instaurer une dynamique entre les différents blocs de sens.

Le deuxième volet a trait à la dimension linguistique du texte, et correspond à l'aspect micro-structurel où sera pris en considération ce qui est fortement codé par la grammaire, ce qui renvoie aux éléments formels de la langue: ponctuation, vocabulaire, conjugaison, grammaire et orthographe. Notre objectif est de vérifier l'état général de la langue utilisée, ainsi que la qualité d'expression des étudiants testés.

Enfin, le dernier outil d'investigation consiste en l'analyse du contenu du tableau récapitulatif dont le choix se justifie par le fait que cette pratique d'élaboration de tableaux cherche notamment à développer une compétence textuelle. L'objectif est d'examiner la capacité d'analyse et de synthèse, vérifier si cette dernière est plus ou moins développée, en comparaison avec celle mise en œuvre pour la rédaction du compte rendu.

\section{III- Présentation des résultats}

Dans ce qui suit, nous présenterons les résultats saillants de la recherche et procéderons à leur interprétation comparative selon les niveaux considérés. Il ressort du questionnaire que les entraînements reçus lors de cette formation permettent d'améliorer les performances des étudiants et ce, pour les différents savoir-faire dispensés, et que l'enseignement $\mathrm{du}$ français fonctionnel contribue au succès de l'apprenant favorisant la construction du savoir au cours de ses études. Par ailleurs, l'utilité de la formation est 
exprimée par l'ensemble des enquêtés, cependant, elle est jugée plus pertinente et opérationnelle par les étudiants du deuxième cycle «cycle d'ingénieur », et s'accentue au fur et à mesure que l'on avance dans les niveaux d'études comme le montrent les pourcentages suivants :

Pour le premier savoir-faire (lecture active), 81,67\% de l'ensemble des étudiants affirment que l'entraînement reçu lors de ce module permet l'amélioration de la performance de lecture. Les moyennes enregistrées pour la variable «utilité » sont légèrement supérieures à la moyenne générale réalisée par l'ensemble de l'échantillon, calculée à $(3,33)$ et jugée positive car dépassant la moyenne de 3 si l'on prend en considération le codage attribué à l'échelle numérique présentée dans le questionnaire. Notons à cet égard que les moyennes des niveaux 3 et 4 sont quasi identiques, respectivement de $(3,40)$ et $(3,41)$ dépassant ainsi celle réalisée par le niveau 2 (3,17). Pour la prise de notes sur document écrit, 76,67\% de l'ensemble des enquêtés affirment avoir développé cette habileté suite à l'entraînement reçu. Le pourcentage le plus élevé est réalisé par le niveau 4, de l'ordre de 90,91\%, suivi par le niveau $3(80 \%)$, et $(55,56 \%)$ enregistré par le niveau 2. Quant à l'utilité du module, la même remarque s'impose en ce sens que les moyennes enregistrées par les niveaux 3 et 4 sont sensiblement supérieures à celles du niveau 2 , respectivement de $(3,60),(3,67)$ et $(3,22)$.

Pour la prise de notes sur document oral, $85 \%$ des enquêtés affirment la contribution de l'entraînement reçu à la réalisation des tâches d'étudiant. Parallèlement, les moyennes réalisées par l'ensemble des étudiants sont positives, présentées comme suit : le niveau 2 $(3,58)$, le niveau $3(4,15)$, le niveau $4(3,56)$. Pour l'exposé, le niveau 4 a réalisé un score de $100 \%$, suivi par le niveau $3(70 \%)$ et en dernière position, nous retrouvons le niveau 2 avec un pourcentage de $(27,78 \%)$. Les étudiants, plus particulièrement ceux des niveaux 3 et 4 affirment avoir développé cette capacité et peuvent réaliser un exposé de manière autonome. Les moyennes enregistrées par l'ensemble des étudiants enquêtés sont supérieures à la moyenne générale, dont celles réalisées par les niveaux 3 et 4 sont quasi identiques, respectivement de $(4,40)$ et $(4,41)$.

En ce qui concerne le dernier module «l'écrit », les pourcentages les plus élevés des étudiants affirmant avoir tiré profit de ces entraînements et par conséquent accru leurs compétences à l'écrit, sont réalisés par les niveaux 3 et 4 , respectivement de $(80 \%)$ et $(72,73$ $\%)$. Tandis que le niveau 2 se positionne à la fin (40\%). Quant à l'utilité de la formation, nous avons noté que la moyenne la plus élevée est réalisée par le niveau 4 (3,90), suivi par le niveau $3(3,80)$ et enfin le niveau $2(3,25)$. Nous n'omettons pas de signaler que les commentaires formulés par les enquêtés confirment l'utilité de la formation et la nécessité 
d'un tel enseignement pour suivre leurs études agronomiques assurées en langue française, et suggèrent par ailleurs d'accorder la priorité à l'écrit.

Les résultats issus de l'analyse du premier paramètre visant à évaluer l'habileté de lecture-compréhension ont pu démontrer que les étudiants, tous niveaux confondus, ont la capacité d'identifier les indices significatifs du texte : iconiques, formels et sémantiques, et que les scores enregistrés correspondent aux stratégies les plus complexes. Ces dernières constituent une passerelle indispensable à l'élaboration du compte rendu. Les résultats issus de l'analyse du deuxième paramètre à savoir la dimension textuelle du compte rendu ont mis en évidence un ensemble de caractéristiques rencontrées chez la majorité des étudiants, schématisées comme suit :

- la présence de structuration textuelle;

- l'adéquation sémantique entre les articulateurs et les contenus qui suivent ;

- l'utilisation correcte des connecteurs ainsi que la maîtrise de leur valeur, élément indispensable à la cohésion interphrastique et à la cohésion textuelle;

- l'adéquation des textes produits au contenu du texte-source ;

- la capacité à hiérarchiser le contenu reproduit ;

- l'emploi des organisateurs méta-textuels (tirets et les deux points) lesquels rendent compte de l'articulation du discours produit ;

- bonne maîtrise du mécanisme de reformulation.

Il va sans dire que l'analyse a fait ressortir notamment des lacunes présentées comme suit :

- l'absence de ponctuation engendrant une mauvaise segmentation des énoncés ;

- la présence de confusions sémantiques et d'interférences avec la langue arabe considérée comme la première langue (L1).

L'analyse de la dimension linguistique des productions écrites a permis de relever une forte concentration des erreurs autour de la catégorie «orthographe » suite au décompte fréquentiel, et sur la base des pourcentages calculés pour chaque catégorie. Ainsi, pour le niveau 2, nous avons relevé $55 \%, 56 \%$ pour le niveau 3 et $60 \%$ enregistré par le niveau 4 . Il importe de signaler que les autres catégories recensées à savoir, la morphosyntaxe, la ponctuation et le lexique ne sont pas de moindre importance, et que les difficultés d'ordre grammatical rencontrées à l'écrit se posent avec acuité même pour les niveaux avancés, notamment la $3^{\mathrm{e}}$ et $4^{\mathrm{e}}$ année.

Enfin, il ressort des résultats du tableau récapitulatif que la capacité d'analyse chez l'étudiant scientifique de l'ENAM est à développer, et que certaines opérations cognitives ne 
sont pas uniquement tributaires de la capacité de compréhension. Cette dernière, à son tour, ne doit pas être approximative mais analytique. En effet, le dépouillement et la schématisation effectués, par la majorité des étudiants, sur le texte ne rendent pas compte de toutes les données et reflétent une compréhension moins nuancée et complexe.

\section{Conclusion et propositions}

L'étude a permis d'apporter quelques éléments de réponse aux questions formulées au début. En effet, nous avons décelé une amélioration des performances langagières et rédactionnelles eu égard aux acquis intégrés lors du cursus de formation en FOS assuré à l'ENAM, surtout chez les étudiants du cycle Ingénieur (les niveaux 3 et 4). Cependant, cette amélioration ne touche pas toutes les habiletés telle l'habileté de synthèse comme il ressort du tableau récapitulatif. Par ailleurs, la nouvelle approche de l'enseignement de la langue française ne peut combler toutes les lacunes, d'ordre linguistique, signalées par les professeurs des sciences, et doter les étudiants de compétences adéquates. A l'égard des résultats enregistrés, nous avons formulé un ensemble de propositions qui pourraient contribuer à l'amélioration du niveau de langue et du profil langagier de l'étudiant scientifique de l'ENAM, répertoriées en trois catégories :

1- Propositions liées aux réajustements estimés judicieux de certains modules de formation, en l'occurrence les modules intitulés «Compréhension écrite analytique » et «Renforcement de la compétence linguistique ». Ces derniers doivent être révisés et intégrés en tant qu'objectifs principaux et non transversaux pour en faire des modules autonomes à partir de la première année d'études.

Par ailleurs, les dits modules pourraient concourir à développer la capacité d'expression et de production écrites chez les étudiants de l'ENAM et ce, en les entraînant à une lecture globale dont les pratiques s'appuieraient sur des phénomènes de discours, c'est-àdire sur le relevé de modèles linguistiques d'organisation textuelle et sémantico-grammaticale afin de les faire systématiser, pour que l'étudiant puisse les identifier et les réemployer, améliorant ainsi sa propre compétence de l'écrit ;

2- Enseigner les techniques d'expression écrite et développer les habiletés de communication écrites qui permettent l'accès aux textes écrits. Ces habiletés se trouvent être indispensables au profil requis pour un étudiant scientifique car elles constituent l'aptitude de base. Cette dernière lui permettra de maitriser un certain nombre de connaissances d'ordre textuel et de techniques indispensables à l'interprétation correcte des documents écrits ;

3- Propositions relatives aux options méthodologiques mises en œuvre actuellement au 
Maroc pour l'enseignement du français ; lesquelles se sont avérées inadéquates. En effet, les résultats enregistrés montrent que la finalité de l'enseignement du français ne saurait se réduire à l'acquisition de compétences méthodologiques, mais doit viser également la maîtrise du système purement linguistique avec ses structures lexicales et phonologiques. Par ailleurs, l'approche fonctionnelle adoptée au Maroc pour l'enseignement du français suite à l'arabisation des matières scientifiques au niveau secondaire, s'est révélée insuffisante car elle doit intégrer d'autres paramètres et prendre en considération l'aspect «langue» pour en faire une priorité au même titre que les autres compétences que l'on cherche à développer, notamment la compétence de communication. Ainsi, l'objectif principal de l'enseignement de la langue française considérée comme la première langue étrangère au Maroc, ne saurait plus se limiter à fournir à l'apprenant un instrument de communication pour qu'il s'en serve dans des situations réelles de communication écrite ou orale, mais considérer la langue à la fois comme un savoir que l'apprenant doit acquérir et un savoir-faire qu'il doit maîtriser en situation.

A cet égard, nous partageons l'avis de certains formateurs et praticiens tels MarieMadeleine Rivenc-Chiclet; Sibylle Bolton; Sophie Moirand, et nous considérons les deux domaines de la compétence (le linguistique et le communicatif) comme s'additionnant et si étroitement solidaires qu'il est impossible de les distinguer. En effet, le reproche qui est fait à l'enseignement de type communicatif concerne les faiblesses en matière phonétique et grammaticale. Aussi pensons-nous qu'il faut prendre en considération la composante linguistique, c'est-à-dire l'habileté qui permet à l'apprenant d'interpréter et d'appliquer les règles de la phonétique, de la phonologie, de la morphosyntaxe et de la sémantique.

Néanmoins, il ne s'agit pas de prévoir un enseignement dichotomique, ou de faire une quelconque distinction entre la compétence linguistique et la compétence communicative, il est nécessaire de n'en négliger aucun. Si la composante linguistique comporte l'apprentissage des structures phonétiques, morphologiques, lexicales et textuelles de la langue, la compétence communicative dans sa globalité, (la composante discursive, référentielle, socioculturelle) se chargera de la connaissance des règles psychologiques, culturelles et sociales qui gèrent l'utilisation de la parole dans un cadre social.

A notre sens, les stratégies de renforcement linguistique destinées au public scientifique marocain ne peuvent dissocier la langue de la communication. La qualité de la syntaxe et la richesse du lexique font partie des contenus que nécessite la communication des savoirs scientifiques. Aussi, l'accent mis sur la communication en situation didactique dans le supérieur n'est-il nullement incompatible avec le rôle primordial de l'école (fondamental et 
secondaire) dans l'acquisition réelle de la maîtrise linguistique qui conditionne tant la réception que la cohérence ou la cohésion des discours scientifiques ou techniques.

\section{Bibliographie}

Adam, Jean-Michel. Eléments de linguistique textuelle, théorie et l'analyse de la linguistique textuelle. Np : Mardaga, 1990.

Bolton, Sibylle. Evaluation de la compétence communicative en langue étrangère. Paris : Hatier-Credif, 1987.

CECRL (Cadre Européen Commun de Référence pour les Langues). Apprendre, enseigner, évaluer. Strasbourg : Division des politiques linguistiques, 2001.

Fontaine, François. Linguistique et analyse textuelle. Paris : PUF, 1980.

Moirand, Sophie. Enseigner à communiquer en langue étrangère. Paris : Hachette, 1982.

Rivenc-Chiclet, Marie-Madeleine. «Approche communicative : effets pervers, paradoxes et contradictions ». Travaux de la didactique du français langue étrangère 9 (1983).

Rück, Heribert. Linguistique textuelle et enseignement du français. Paris : Hatier-Credif, 1980.

Tamesna, Jamila. «Quelques aspects de la problématique de l'enseignement du français de spécialité sur objectifs spécifiques». Recherches pédagogiques. Revue de l'Association Marocaine des Enseignants de Français 3 (1993) : 31-41.

---. «Etude de l'impact de l'enseignement du français fonctionnel sur la compétence textuelle réceptive et productive des étudiants scientifiques arabisés : cas de 1'Ecole Nationale d'Agriculture de Meknès ». Doctorat d'Etat en Didactique du Français soutenu à la Faculté des Sciences de 1'Education, Rabat, 2002.

Van Dijk, Teun. «Grammaires textuelles et structures narratives ». Sémiotique narrative et textuelle. Paris : Larousse, 1973. 177-207. 